\title{
An efficient method for eccrine gland isolation from human scalp
}

DOI:

10.1111/exd.13505

\section{Document Version}

Accepted author manuscript

Link to publication record in Manchester Research Explorer

\section{Citation for published version (APA):}

Jimenez, F., Alam, M., Hernandez, I., Poblet, E., Hardman, J., \& Paus, R. (2018). An efficient method for eccrine gland isolation from human scalp. Experimental Dermatology, 27(6), 678-681. https://doi.org/10.1111/exd.13505

\section{Published in:}

Experimental Dermatology

\section{Citing this paper}

Please note that where the full-text provided on Manchester Research Explorer is the Author Accepted Manuscript or Proof version this may differ from the final Published version. If citing, it is advised that you check and use the publisher's definitive version.

\section{General rights}

Copyright and moral rights for the publications made accessible in the Research Explorer are retained by the authors and/or other copyright owners and it is a condition of accessing publications that users recognise and abide by the legal requirements associated with these rights.

\section{Takedown policy}

If you believe that this document breaches copyright please refer to the University of Manchester's Takedown Procedures [http://man.ac.uk/04Y6Bo] or contact uml.scholarlycommunications@manchester.ac.uk providing relevant details, so we can investigate your claim.

\section{OPEN ACCESS}




\section{Experimental Dermatology}

\section{An efficient method for eccrine gland isolation from human scalp}

\begin{tabular}{|r|l|}
\hline Journal: & Experimental Dermatology \\
\hline Manuscript ID & EXD-17-0449.R1 \\
\hline Date Submitted by the Author: & 19-Jan-2018 \\
\hline Complete List of Authors: & $\begin{array}{l}\text { Jimenez, Francisco; Mediteknia Hair Transplant Clinic; Universidad } \\
\text { Fernando Pessoa Canarias; Grupo de Patología Medica, IUIBS, Universidad } \\
\text { de Las Palmas de Gran Canaria } \\
\text { Alam, Majid; Mediteknia Hair Transplant Clinic; Universidad Fernando } \\
\text { Pessoa Canarias; Monasterium Laboratory } \\
\text { Hernandez, Irene; Mediteknia Hair Transplant Clinic } \\
\text { Poblet, Enrique; Univ Murcia, Pathology; University General Hospital, } \\
\text { Pathology } \\
\text { Hardman, Jonathan; University of Manchester, Institute of Inflammation } \\
\text { and Repair } \\
\text { Paus, Ralf; University of Manchester, Institute of Inflammation and Repair; } \\
\text { Monasterium Laboratory }\end{array}$ \\
\hline Keywords: & \begin{tabular}{l} 
Eccrine glands, Sweat glands, Hair Follicle, Follicular Unit, Methylene Blue \\
\hline
\end{tabular} \\
\hline $\begin{array}{l}\text { Note: The following files were submitted by the author for peer review, but cannot be converted to } \\
\text { PDF. You must view these files (e.g. movies) online. }\end{array}$ \\
\hline My Movie.mp4
\end{tabular}


Type of article: Methods Letter to the Editor

\title{
An efficient method for eccrine gland isolation from human scalp
}

\author{
Jimenez $F^{1,2,3}$, Alam $M^{1,2,4}$, Hernandez $I^{1}$, Poblet $E^{5}$, Hardman $J A^{6}$, Paus ${ }^{6}$ \\ Mediteknia Hair Transplant Clinic and Hair Lab ${ }^{1}$, Gran Canaria, Canary Islands, Spain \\ Universidad Fernando Pessoa Canarias ${ }^{2}$, Gran Canaria, Spain \\ Medical Pathology Group, IUIBS, Universidad de Las Palmas Gran Canaria ${ }^{3}$ \\ Monasterium Laboratory, Münster, Germany ${ }^{4}$ \\ Reina Sofia University General Hospital and University of Murcia ${ }^{5}$, Spain \\ The Centre for Dermatology Research, University of Manchester, MAHSC, and National Institutes of \\ Health Biomedical Research Center, Manchester, $U K^{6}$
}

\section{Author for Correspondence and Reprint Requests:}

Francisco Jimenez, MD (Mediteknia Clinic)

Av. Alcalde Ramirez Bethencourt, 20

Las Palmas de Gran Canaria 35004

Canary Islands, Spain

Email: fjimenez@mediteknia.com

Phone: +34-928232278

Number of words: 996

References: 24

Figures: 2

Supplementary Material: Text (348 words), Figures (2), Video (1)

Keywords: Eccrine glands, sweat glands, hair follicle, hair transplantation, hair graft,

follicular unit, sweat, neutral red, methylene blue 


\begin{abstract}
:
We describe a simple and efficient method to isolate eccrine sweat glands from the human scalp. This method is inspired by the hair graft harvesting method used in hair transplantation. Based on the recently described anatomical relationship between the scalp hair follicle and the eccrine gland, we have found that scalp follicular unit grafts are an excellent eccrine gland isolation source, especially for the coiled component. In order to make the gland visible for stereoscopic microdissection, the follicular units need to be previously stained with a vital dye like Methylene Blue or Neutral Red. The simplicity and efficiency of this isolation method should encourage further research into human eccrine sweat gland function which has always been hindered by the difficulty of gland isolation.
\end{abstract}




\section{Background}

Eccrine glands (EGs) are cutaneous appendages that produce sweat and act as our primary source of cooling. Besides their critical role in thermoregulation, they also play a less understood yet important role in cutaneous wound healing (1-4). Research into human eccrine sweat gland function has been greatly hindered by problems of gland isolation (supplementary text). Obtaining full intact EGs by stereoscopic microdissection of human skin is a very difficult, laborious and low yieldgiving technique (5-11). Although other isolation methods such as enzymatic collagenase digestion do yield a larger number of glands, exposure to enzymatic digestion may be potentially damaging for physiological functional studies (12-15). In this article, we describe an efficient method of isolating human eccrine sweat glands from follicular unit grafts harvested from the scalp. The idea of harvesting EGs from the scalp arose originally after our reported observation of the striking and consistent anatomical association between eccrine coils and hair follicles (HF) (16).

\section{Question Addressed}

Given the intimate association between the EG and HF, can eccrine coils be successfully isolated from human follicular unit (FU) hair transplant grafts?

\section{Experimental Design}

Our method of EG isolation is based on two premises: 1) due to the reported anatomic association between EGs and scalp HFs, we thought that an excellent and readily available source to obtain EGs was from FU grafts harvested in hair transplant 
procedures, and 2) in order to isolate the EGs by stereoscopic microdissection, a supravital dye could be used to make them visible under a stereomicroscope. Different supravital dyes to highlight the EGs were tried, including Methylene Blue (MB), and Neutral Red $(N R)(6,17,18)$. All FUs were donated by patients undergoing hair transplant surgery for androgenetic alopecia after giving written informed consent. As a reminder, the FU is the prime element used in modern hair transplantation (19). An FU is a histological structure composed of 1 to 4 terminal hair follicles along with their sebaceous glands and arrector pili muscles (20). FUs can be obtained in hair transplantation in two ways: 1) by directly excising individual FUs using small round punches ( 0.8 to $1.00 \mathrm{~mm}$ in diameter), a technique known as FUE (Follicular Unit Extraction) (21), or 2) by stereomicroscope dissection of small slivers sectioned from a donor strip (strip harvesting technique) . In the FUE technique, the punch is introduced to a depth of around $3 \mathrm{~mm}$, and the $\mathrm{FU}$ is then released from the subcutaneous tissue with fine tip forceps (supplementary video) (Fig 1). It is important to have the skill to insert the punch following the hair shaft angle in order to avoid hair follicle transection.

\section{Results}

$M B$ and NR gave a very clear delineation of the $E G$, providing a distinct blue and red coloring of the EG, respectively, easily visible against the background of the dermal collagen. We found that immersing the FU in a few drops of $\mathrm{MB}$ at a concentration of $0.02 \%$ or in NR at a concentration of $0.2 \%$ in physiologic saline for $10-15$ minutes was sufficient to highlight the EGs (Figure 2 \& Supp. Fig 2). 
EG isolation can be accomplished using either of the two different FU graft harvesting methods: strip harvesting and FUE. Using the strip harvesting technique, we observed that the small vertical slivers in which the long strip is divided - of 1 to 2 FU thickness contain numerous EGs that can be easily identified by MB or NR staining and later microdissected (Fig. 2 A,B).

In our experience, EG isolation was even easier when using FU grafts harvested with FUE punches, because the small punches used to excise the FUs, left minimal dermal surrounding tissue, making EG coil dissection faster since the coil was not attached to dermal fibers (Fig. 2 C,D). Anatomically, the EGs are always embedded in adipocytes (dermal fat tissue) and located at a depth of 2 to $3.5 \mathrm{~mm}$ below the epidermal surface of human scalp, at the inferior portion of anagen terminal hair follicles, and always below the sebaceous gland and arrector pili muscle (Suppl Fig. 1). We noted that the smaller the caliber of the punch used in FUE, the higher the risk of transecting and damaging the EG coil. For this reason, we believe that the use of $1 \mathrm{~mm}$ diameter punches is ideal for this purpose. In addition, the ductal excretory portion cannot be isolated with this method because at some point the duct is transected by the punch as depicted by the schematic in figure 1.

Although the main goal of this study is to describe a rapid and efficient source for EG isolation, ex vivo culture of isolated EGs in a mixture of supplemented Williams E and F12 media, demonstrated that they are still viable after 6 days as shown by Neutral Red uptake and Calcein AM $(2 \mu \mathrm{M})$ (ThermoFisher Scientific), a cell-permeant dye that in live cells is converted to green-fluorescent calcein (Supp. Fig. 2). 


\section{Conclusion}

With the emerging discovery of the importance of the EG in roles other than sweating (including, amongst others, wound healing), an EG isolation method is required that is simple, efficient, and that can also be used with living tissue to maintain its morphologic integrity.

Researchers should consider scalp FU grafts obtained from hair transplant procedures as an efficient source for isolating human EGs. Moreover, once the eccrine glands have been dissected from the FUs, the hair follicle grafts could still be used for hair transplantation, so no human tissue is wasted.

Isolation of human EGs ex vivo that maintain their morphological integrity and function can also be an invaluable source for the cosmetic industry interested in studying the sweat response to pharmacological products in healthy as well as in pathologic disorders (e.g. anhidrosis, hypo and hyperhidrosis). As a further fascinating challenge, the possibility exists of investigating the clinical outcome after transplanting exclusively EGs in special clinical situations, for example to extensive deep-burn survivors whose scarring skin covered with skin grafts is devoid of sweat glands and suffers from heat intolerance (22-24). 


\section{References:}

1. Lu C, Fuchs E. Sweat gland progenitors in development, homeostasis, and wound repair. Cold Spring Harb Perspect Med 2014; 4: a0152223.

2. Sato K, Kand WH, Saga K, Sato KI. Biology of sweat glands and their disorders. I. Normal sweat gland function. J Am Acad Dermatol 1989; 20:537-63.

3. Rittié L, Sachs DL, Orringer JS, Voorhees JJ, Fisher GJ. Eccrine sweat glands are major contributors to reepithelialization of human wounds. Am J Pathol 2013; 182: 163.

4. Pontiggia L, Biedermann T, Böttcher-Haberzeth S, Oliveira C, Braziulis E, Klar AS, Meuli-Simmen C, Meuli M, Reichmann. De novo epidermal regeneration using human eccrine sweat gland cells: higher competence of secretory over absorptive cells. J Invest Dermatol 2014; 134: 1735-42.

5. Sato K, Sato F. Individual variations in structure and function of human eccrine sweat gland. Am J Physiol 1983; 245: R203-8.

6. Wolfe S, Cage G, Epstein M, Tice L, Miller H, Gordon RS Jr. Metabolic studies of isolated human eccrine sweat glands. J Clin Invest 1970; 49: 1880.

7. Mangos J. Transductal fluxes of $\mathrm{Na}, \mathrm{K}$, and water in the human eccrine sweat gland. Am J Physiol 1973; 224: 1235-40.

8. Lee $\mathrm{CM}$, Jones $\mathrm{CJ}$, Kealey $\mathrm{T}$. Biochemical and ultrastructural studies of human eccrine sweat glands isolated by shearing and maintained for seven days. J Cell Sci 1984; 72: 259. 
9. Kurata R, Futaki S, Nakano I, Tanemura A, Murota H, Katayama I, Sekiguchi K. Isolation and characterization of sweat gland myoepithelial cells from human skin. Cell Struct Func 2014; 39: 101.

10. Lee CM, Carpenter F, Coaker T, Kealey T. The primary culture of epithelia from the secretory coil and collecting duct of normal human and cystic fibrotic eccrine sweat glands. J Cell Sci 1986; 83: 103-18.

11. Kurata R, Futaki S, Nakano I, Fujita F, Tanemura A, Murota H, Katayama I, Okada F, Sekiguchi K. Three-dimensional cell shapes and arrangements in human sweat glands as revealed by whole-mount immunostaining. PLoS ONE 2017; 12: e0178709.

12. Okada N, Kitano Y, Morimoto T. Isolation of a viable eccrine sweat gland by dispase. Arch Dermatol Res 1983; 275: 130-3.

13. Kealy T. The metabolism and hormonal responses of human eccrine sweat glands isolated by collagenase digestion. Biochem J 1983; 212: 143-8.

14. Gao Y, Li M, Zhang X, Bai T, Chi G, Liu JY, Li Y. Isolation, culture and phenotypic characterization of human sweat gland epithelial cells. Int J Mol Med 2014; 34:997.

15. Hubka KM, Wu D, Harrington DA, Langer JC, Pocard T, Jammayraco O, FarachCarson MC, Pradhan-Bhatt S. Dissociative and nondissociateve models for culture of human eccrine glands for toxicology testing and tissue engineering applications. Applied in Vitro Toxicol 2015; 1: 187-97.

16. Poblet E, Jiménez-Acosta F, Hardman JA, Escario E, Paus R. Is the eccrine gland an integral, functionally important component of the human scalp pilosebaceous unit? Exp Dermatol 2016; 25: 149. 
17. Hurley HJ, Witkowski J. Dye clearance and eccrine sweat secretion in human skin. J Invest Dermatol 1961; 36: 259.

18. Brayden DJ, Fitzpatrick J. Cultured human sweat gland epithelia. Isolation of glands using Neutral Red. Pharm Res 1995; 12: 171.

19. Vogel JE, Jimenez F, Cole J, Keene SA, Harris JA, Barrera A, Rose PT. Hair restoration surgery: the state of the art. Aesthet Surg J 2013; 33: 128.

20. Poblet $E$, Jimenez F, Ortega F. The contribution of the arrector pili muscle and sebaceous glands to the folicular unit structure. J Am Acad Dermatol 2004; 51: 217.

21. Jiménez-Acosta F, Ponce-Rodríguez I. Follicular unit extraction for hair transplantation: an update. Actas Dermosifiliogr 2017; 108: 532.

22. Ma K, Tan Z, Zhang C, Fu X. Mesenchymal stem cells for sweat gland regeneration after burns: from possibility to reality. Burns 2016; 492-9.

23. Pontiggia L. Eccrine sweat gland regeneration: still a story of "blood, toil, tears, and sweat". Br J Dermatol 2017; 176: 1425

24. Klaka P, Grüdi S, Banowski B, Giesen M, Sättler A, Proksch P, Weiss T, Förster T. A novel organotypic 3D sweat gland model with physiological functionality. PLoS ONE 12(8): e0182752. 


\section{Figure Legends}

Figure 1. Schematic of eccrine gland isolation technique using an FUE punch.

This figure illustrates the technical procedure of eccrine gland isolation from the scalp follicular unit with a circular micro-punch (FUE technique). The left drawing depicts the anatomy of a scalp follicular unit (FU), showing the location of the eccrine coil in relation to the anagen terminal hair follicles, sebaceous glands and the arrector pili muscles. Note that most human scalp FUs contain 2 or 3 terminal hair follicles. The eccrine coil is embedded in adipocytes (dermal fat tissue). The arrector pili muscles that emerge from each of the follicles that form the FU join together forming a single muscular bundle. The middle drawing shows how the punch needs to be introduced into the scalp skin with the angle of the punch parallel to the hair shaft direction in order to avoid FU transection. The penetration of the punch to a depth of 3-4 $\mathrm{mm}$ is sufficient with most patients to release the FU from the surrounding dermal collagen attachments and allow it to then be easily removed with fine tip forceps (drawing on the right).

Figure 2. Methylene Blue allows easy identification of human eccrine glands in scalp follicular units. A typical thin vertical sliver of scalp skin dissected under the stereomicroscope during a strip harvesting hair transplant procedure unstained $(A)$ and stained with Methylene Blue (B). Note the position of the eccrine coils, in the deep dermis, approximately 2-3.5 $\mathrm{mm}$ beneath the epidermis and embedded in adipocytes. A three-hair follicular unit (harvested with a $1 \mathrm{~mm}$ punch) stained with Methylene Blue with two eccrine coils between the hair follicles (C) and the eccrine coils after dissection from the follicles (D). 

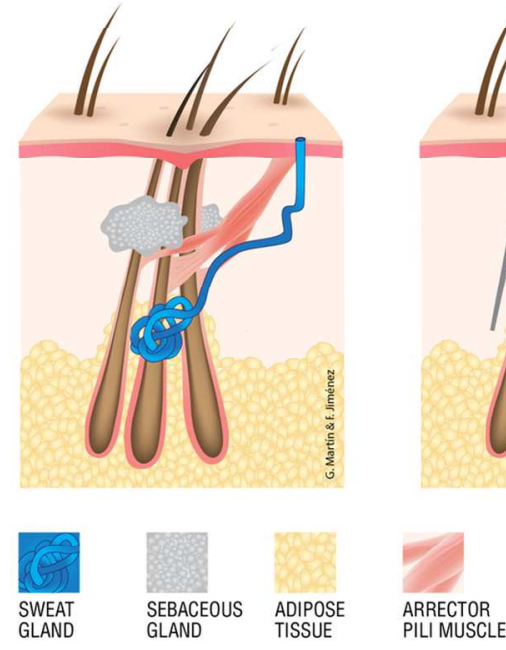

$229 \times 205 \mathrm{~mm}(150 \times 150 \mathrm{DPI})$

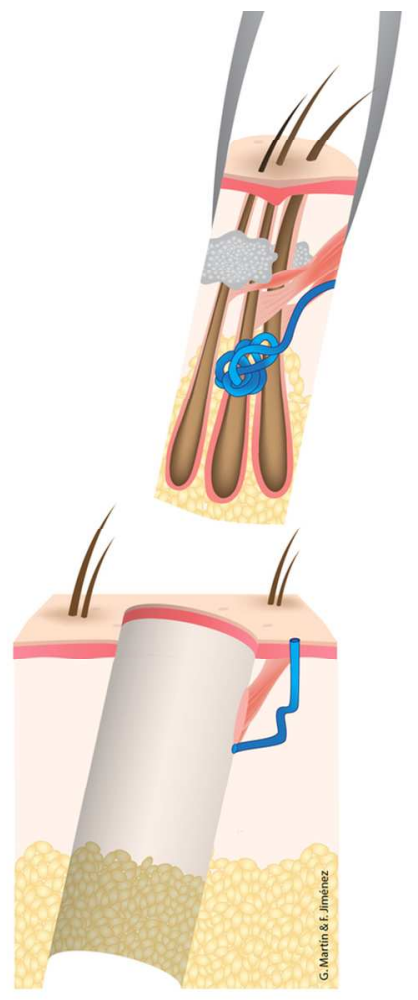

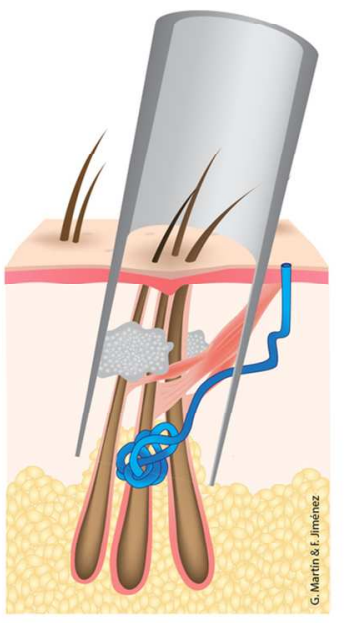



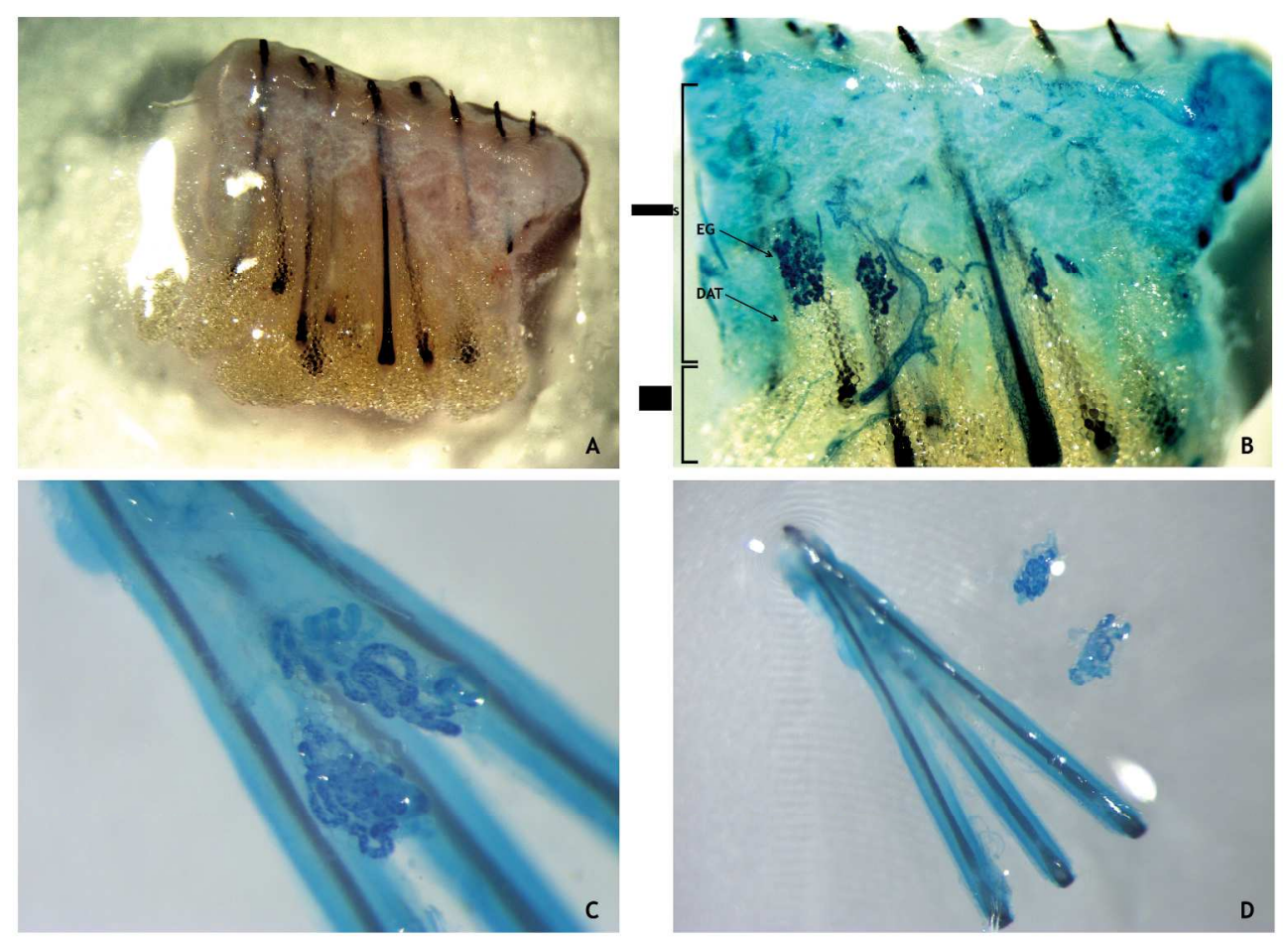

1091×800mm (72 x 72 DPI) 


\section{Supplementary text:}

\section{Methods of eccrine gland isolation:}

Sweat gland research is still rather slow in comparison with other fields, with one of the main reasons being the difficulties in isolating it from human skin. Microdissection has been tried, but this is an extremely difficult technique which gives a low yield (511). Sato (7) isolated sweat glands from $1 \mathrm{~cm} \times 0.5 \mathrm{~cm}$ ellipse skin from the forearms, under a stereomicroscope, by gently teasing away periglandular collagen fibers with sharp forceps. The method is very difficult and time-consuming and has a low efficiency. Lee et al (10) and Brayden et al (20) described a better way to isolate human sweat glands by chopping skin samples with sharp scissors. The principle was that sweat glands "pop out" from surrounding collagen and fat due to shearing forces created by the chopping.

Another way to isolate glands is by enzymatic digestion of skin samples with collagenase. This method involves overnight incubation of skin samples with collagenase. It yields a larger number of glands (14-16), but the exposure of cells to collagenase is potentially damaging.

One of the difficulties encountered with EG isolation by microdissection is that EGs, as opposed to sebaceous glands or hair follicles, are not visible under the stereomicroscope, which makes dissection impossible. To circumvent this problem, Wolfe (8) and Mangos (9) stained the gland prior to dissection with the supravital dye Methylene Blue. This dye can be introduced in the skin without damaging the cells. It has been used in the past to study eccrine sweat gland function by intradermal injection and evaluation of the blue sweat droplets excreted at the ductal orifices (19). 
Another vital dye used to stain eccrine glands is Neutral Red (20). Neutral red is actively secreted into the lumen of the duct through organic ion pathways. According to Brayden (20), Neutral Red staining allowed on average $52 \%$ more sweat glands to be isolated from the chopped suspension of human skin. The Neutral Red staining method has been shown to be non-toxic at low concentration and the stained glands grow in culture just as successfully as controls.

\title{
Supplementary Figures:
}

Supplementary Figure 1.

Eccrine glands are clearly visualised after follicular unit grafts are stained with Neutral Red and Methylene Blue

Follicular units harvested from scalp skin with a $1 \mathrm{~mm}$ punch and stained for $10-15$ minutes with (a) Neutral Red (0.2\%) and (b) Methylene blue $(0.02 \%)$ provides a very clear intravital identification of the eccrine glands (arrows), which are normally not visible under the stereomicroscope.

\author{
Supplementary Figure 2 \\ Eccrine glands are still viable after 6 days of ex vivo culture \\ After 6 days of culture in a mixture of supplemented Williams E and F12 media, eccrine \\ glands were stained with two markers to assess their viability: Neutral Red (a) which
}


stains lysosomes of live cells, and (b) Calcein AM, a cell-permeant dye which in live cells the non-fluorescent calcein AM is converted to green-fluorescent calcein.

\section{Supplementary Video:}

Follicular unit extraction: Surgical technique using a motorized $1 \mathrm{~mm}$ punch for harvesting follicular units from the scalp. These FUs can be stained with Neutral Red or Methylene Blue for eccrine gland identification under the stereomicroscope. 
Eccrine glands are clearly visualised after follicular unit grafts are stained with Neutral Red and Methylene Blue

$190 \times 254 \mathrm{~mm}(96 \times 96$ DPI) 
a

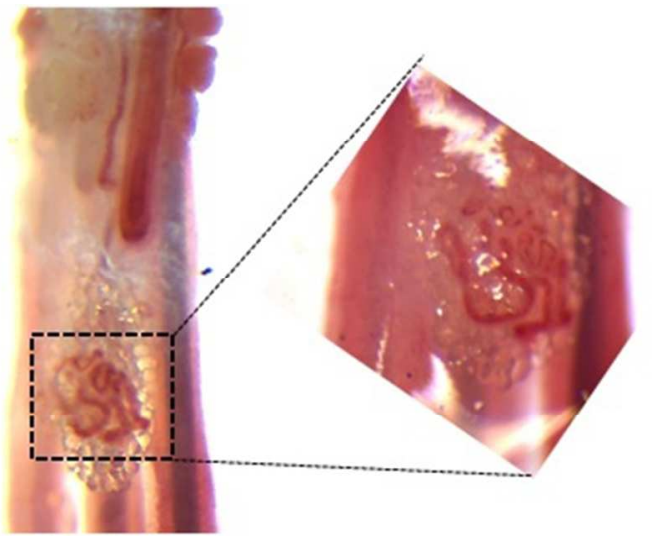

Eccrine glands are still viable after 6 days of ex vivo culture

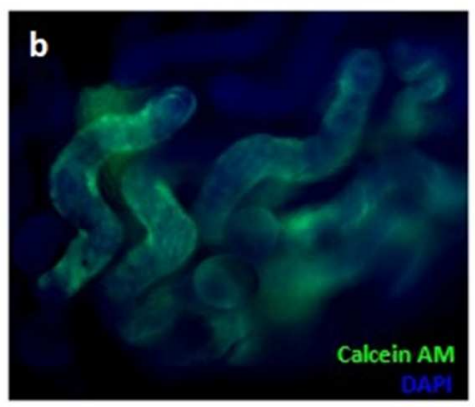

$176 \times 74 \mathrm{~mm}(96 \times 96$ DPI) 
Author's Response to Decision Letter for (EXD-17-0449)

An efficient method for eccrine gland isolation from human scalp

Dear Professor Luger,

We would like to thank the reviewers for their kind comments and helpful insight/suggestions. We have addressed the reviewers' questions as following:

Reviewer 1:

1. How long can the authors culture the eccrine glands after they isolate eccrine glands?

We have the shown that eccrine glands maintain viability after 6 days of culture as assessed by uptake of the cell viability markers Neutral Red and Calcein AM. We have added this information to the supplementary text of the manuscript and supplementary figure 2 .

2. What kind of culture medium should be used for eccrine gland organ culture?

A mixture of supplemented Williams E and F12 media was used to culture eccrine glands. This has been added to the main text.

3. Could the authors more specify the possible further experiments which can be performed with this isolation technique?

Further experiments could be to utilise this method to test the functionality of eccrine gland i.e. by stimulating with known sweat inducers such as Acetylcholine, as done in a previous study by Sato et al. in the 70s (Sato el al. 1973, American Journal of Physiology) using eccrine glands from monkeys and more recently in 3D organotypic models of eccrine glands (Klaka et al. 2017, Plos One). We could also do the opposite, i.e. to inhibit the sweat production by treating with for example botulinum toxin.

Reviewer: 2

Suggestions for Authors 
This letter by Jimenez and colleagues describes a method for the isolation of eccrine sweat glands from the human scalp. The method is actually not new, as it consists essentially of already established procedures (punches or the strip harvesting technique). Nevertheless, considering the increased interest in eccrine gland research and the lack of simple and effective methods for their isolation, this is an important study. What a terrific movie!

1) The authors repeatedly mention what seems to be a limitation of the method: the fact that only the coiled compartment of the gland can be isolated, while the ductal secretory portion gets lost. However, what does this limitation exactly mean? What kind of basic science or translational studies are handicapped by the loss of this particular anatomical part of the gland?

From a functional point of view, since the coiled gland is the main component of the eccrine gland where the sweat is produced, the loss of the 'straight' ductal portion does not pose a great limitation to further investigation in the context of sweat production. We feel that the main limitation of transecting the sweat ducts would be in studying the role of sweat glands in skin wound healing, since it has been shown that there is a rapid proliferation of the sweat duct epithelium after superficial injuries (Rittie et al. Am J Pathol 2013).

2) Figure identification and labelling need to be significantly improved. For instance, on page 5, line 39, Fig. 1c,d are indicated, while there are not such labels in the figure or in its legend. Are there 3 or 4 suppl. figures? Suppl. Figures need better legends with improved description.

Thank you for highlighting this, it has been corrected with the previous supplementary figures 1 \& 2 combined into one figure and more descriptive legends have been added. 